\title{
Etimologia dei verbi di origine istroveneta nella parlata ciacava di Baratto di Canfanaro
}

\author{
Izvorni znanstveni rad \\ Original scientific paper
}

UDK 811.131.1'373.6(497.5 Barat)

Il lavoro è dedicato all'analisi etimologica e lessicologica dei prestiti istroveneti presenti nella parlata ciacava di Baratto di Canfanaro. L'uso dei romanismi analizzati è stato confermato attraverso la ricerca sul terreno effettuata nel 2012. II lavoro rappresenta l'analisi dei prestiti verbali, di cui è stata individuata l'etimologia prossima e quella remota, consultando i maggiori dizionari etimologici dell'italiano, del croato e delle lingue romanze. Vengono inoltre descritti i cambiamenti fonologici e morfologici subiti dai verbi nel passaggio dall'istroveneto al ciacavo.

Parole chiave: etimologia, romanismi, ciacavo, istroveneto, Baratto (Canfanaro)

\section{Introduzione}

I processo etimologico è un processo di scoperta che presume una ricerca a catena, ovvero stabilire l'etimologia prossima e quella remota di una parola. La prima riguarda la scoperta della lingua (nel nostro caso l'istroveneto) dalla quale una parola viene presa e introdotta nella lingua ricevente (il ciacavo). Ciò può succedere solo attraverso un contatto diretto tra le due lingue. Con etimologia remota si intende la scoperta dell'origine più profonda della parola, ovvero lo strato latino all'interno dell'etimologia romanza. Cogliere l'origine ultima di una forma non è di primaria importanza per lo studioso di lingue romanze, al quale basta riuscire a ricondurre i rapporti genetici di una parola fino ad una determinata epoca anteriore, ovvero lo strato latino, senza andare fino alla radice indoeuropea (Pfister 2001: 36). Il lavoro è incentrato su una piccola parte dell'elemento romanzo presente nella parlata ciacava di Baratto di Canfanaro, ovvero l'analisi etimologica dei prestiti verbali.

I romanismi secondo la definizione di Simeon (1969: 317) sono elementi linguistici appartenenti ad una lingua o dialetto romanzo che entrano in una lingua non romanza. II lemma ciacavo takàt < tacàr rappresenta un italianismo (venezianismo) per il ciacavo, anche se questo lessema entra nell'italiano attraverso il francese, ed è quindi un francesismo per I'italiano. Muljačić (Muljačić in Gačić 1979: 4) dice che quello che più conta nello studio etimologico in funzione lessicologica è di poter individuare con certezza l'ultima lingua fonte da cui s'irradiò un accatto di un'altra lingua.

Il numero dei romanismi penetrati nella parlata ciacava attraverso l'istroveneto è notevole. Questi contatti linguistici sono il risultato di contatti storici e culturali, e quelli 
croato-italiani durano dalle origini della storia croata ${ }^{1}$. II contatto linguistico fa parte di quei processi etnoculturali che si sviluppano laddove su un unico territorio convivono diverse etnie, culture e lingue ${ }^{2}$. Un esempio è proprio l'Istria, luogo di contatto linguistico, e non solo, tra ciacavo, veneziano, istroveneto e istroromanzo. Un'attenzione particolare nello studio delle lingue in contatto va data proprio al contatto tra quelle lingue che non sono geneticamente imparentate. Le influenze reciproche nel contatto linguistico sono sempre legittime e inevitabili. II tipo di contatto che esiste fra ciacavo e istroveneto è quello intimo ${ }^{3}$. Il contatto intimo avviene attraverso un contatto diretto tra i parlanti delle due lingue; vengono inserite parole che hanno già un equivalente nella lingua ricevente. La lingua dominante diventa la fonte da cui attinge l'altra lingua, che ha ovviamente uno status inferiore (Sočanac 2001: 78).

Il dialetto ciacavo cambia da una parte del litorale croato all'altra, e la nostra attenzione si è concentrata su una zona specifica, Baratto di Canfanaro. A differenza della città, la campagna ha un carattere conservativo, una composizione della popolazione più stabile, ed è un ambiente meno influenzabile da elementi esterni. Rappresenta quindi una struttura sociale, etnica e linguistica più favorevole da analizzare (Gačić 1979: 6). II paese di Baratto è posizionato nella parte centrale dell'Istria, fa parte del Comune di Canfanaro, e conta 59 abitanti ${ }^{4}$. Una datazione precisa della nascita dei paesi situati nel Canal di Lemme, e quindi anche di Baratto, non è possibile a causa della carenza di testi storici. Possiamo però asserire che nel Cinquecento il paese di Baratto esisteva già, in base a un testo risalente al 1566 (Bertoša 1995: 155). Seppur non si ha un abitante di nazionalità italiana dal 1880 al 1945, anno in cui Baratto contava addirittura 280 abitanti (Bratulić 1985: 299), la parlata di Baratto conserva un notevole numero di romanismi, penetrati dall'istroveneto.

La parlata di Baratto appartiene al dialetto occidentale icavo-ciacavo, che ha come caratteristica principale il regolare sviluppo del praslavo $e$ in $i$, come negli esempi dica, lipo, siditi, vrime, in cui ci sono anche elementi ecavi come delo, sused. Alcune caratteristiche che possiamo notare nel dialetto icavo-ciacavo (Lisac 2009: 51-55) sono il dittongamento di $e$ ed $o$ lunghe (siestra, piet, vuoda, uovce, guoriti, gruoblje); il passaggio di $o$ atona in $u$ (uženjena) e della o atona in a (matika); la caduta della i davanti a z (zgleda, zgubljen, zgorilo); il cambiamento della preposizione $s$ "con" in z con lo strumentale ( $z$ auton); il passaggio della $m$ in $n$ nelle desinenze e nelle parole invariabili (vidin, plaćan, moren, ustanen); la semplificazione di gruppi consonantici: $p t>t$ (tič), $p \check{s}>\check{s}$ (šenica), $d n>n$ (jeno); l'aggiunta della $j$ a inizio parola (jopet, jima).

I romanismi penetrati nel ciacavo di Baratto sono tutti, come si vedrà più avanti, di origine istroveneta, che è la variante istriana del dialetto veneziano. L'istroveneto ebbe un forte influsso non solo sui dialetti ciacavi, ma anche sulle parlate istriote, che rappresentano lo strato autoctono romanzo preveneziano sviluppatosi dal latino volgare. Parlato in tutte le zone e conosciuto dalla maggioranza della comunità linguistica, l'istroveneto era la lingua comune di commercio e comunicazione, una specie di koiné fino al 1945. Con il cambiamento dei confini statali e le migrazioni interne dopo il grande esodo, l'istroveneto è stato sostituito

I contatti croato-romanzi iniziano nel XI secolo; nel Trecento Venezia prende il controllo amministrativo dell'Istria, e l'istroveneto diventa lingua comune o koiné della penisola, fino a quando non viene soppiantato definitivamente dal croato, nel Novecento.

2 Fu Weinreich il primo a collegare gli elementi linguistici e quelli culturali in una situazione di contatto (Weinreich 2008).

3 Bloomfield descrive per la prima volta i diversi tipi di contatto linguistico nel suo Language.

4 Secondo i risultati dell'ultimo censimento della popolazione del 2011. Il dato è stato preso dal sito internet ufficiale dell'Istituto nazionale di statistica croato (Državni zavod za statistiku): www.dzs.hr (25.10.2012). 
dal croato e dagli idiomi ciacavi dell'Istria croata e da quelli sloveni nell'Istria slovena. Non è cambiato niente invece nell'Istria italiana (Filipi 1996: 120).

\section{Adattamento morfologico dei verbi}

L'adattamento morfologico può essere primario e secondario. I cambiamenti primari avvengono nel processo di assimilazione di un prestito al sistema morfologico della lingua ricevente. In questa fase ci sono tre tipi di trasmorfemizzazione: la sostituzione di grado zero, quella parziale, e la sostituzione completa (Filipović 1986: 119). I prestiti verbali subiscono il processo di trasmorfemizzazione completa ${ }^{5}$, sostituendo la desinenza dell'infinito italiano con quella croata. II cambiamento della desinenza è obbligatorio, affinché il verbo funzioni nel sistema morfologico della lingua ricevente.

Nel ciacavo di Baratto l'infinito termina in -ti (kapiti, disturbati, finiti, partiti, tornati), meno frequentemente in -t (kumpanat, takat). I verbi italiani di I coniugazione (-are) nel passaggio al ciacavo ricevono la desinenza -at(i), mentre quelli di II (-ere) e III (-ire) la desinenza -it(i). Le eccezioni sono rare (metati<meter<mettere; parati<parer<parere; zaskuriti<oscurare).

Il sistema morfologico croato differisce da quello italiano nell'aspetto verbale. Nella lingua italiana I'aspetto del verbo viene definito dalla costruzione della frase e dal tempo usato. Nel croato invece la maggior parte dei verbi è caratterizzata da un aspetto, ovvero le forme morfologiche dei due aspetti dello stesso verbo (svršeni i nesvršeni) sono differenti. Nel processo di assimilazione dei prestiti verbali sorge quindi il problema dell'adattamento dell'aspetto verbale. Di conseguenza possiamo dividere questi prestiti in due gruppi: quelli che esprimono l'aspetto tramite il contesto, senza un segno morfologico, e quelli che lo indicano tramite prefisso o infisso. Sočanac (2004: 166) nota che nel dialetto ragusano l'aspetto viene indicato con l'infisso - $v a$-. Si è notata la stessa caratteristica nella parlata di Baratto con l'esempio gambivati: che esprime un'azione che si ripete nel tempo, a differenza di gambjâti<gambiàr<cambiare. II ragionamento che tutti i prestiti verbali appartengano al gruppo con desinenza -at(i) e -it(i), forse può essere collegato con la possibilità di marcare l'aspetto di ogni verbo con l'infisso -va-. Questo cambiamento avviene nella fase dell'adattamento secondario (Sočanac 2002: 135), che presume una completa integrazione nel sistema ricevente, in cui i prestiti vengono coinvolti nella formazione delle parole tramite

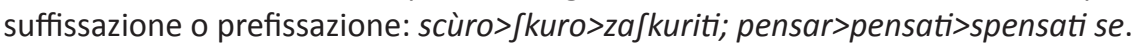

\section{Analisi lessicologica ed etimologica}

Per l'analisi etimologica è stato scelto il gruppo grammaticale dei verbi, usati per arricchire o coprire spazi semantici vuoti nella lingua ricevente, la parlata di Baratto. Per la raccolta dei dati sono stati registrati dei dialoghi con informatori di età avanzata, siccome si presume che un parlante anziano conservi la parlata autoctona. I parlanti in questione non sono bilingui, e utilizzano quindi elementi romanzi, pur non conoscendo l'istroveneto o l'italiano.

Ogni parola è stata analizzata per identificarne l'etimologia prossima e remota (Muljačić 2003: 99). Per trovare l'etimologia prossima sono stati consultati i dizionari dell'istroveneto (Glavinić 2000; Rosamani 1990), del triestino (Doria 1987), e del veneziano (Boerio 1829), mentre per l'etimologia remota i dizionari etimologici della lingua italiana (Battisti 1975;

La sostituzione completa avviene quando i morfemi legati della lingua donatrice vengono sostituiti con quelli della lingua ricevente (Sočanac 2002: 134). 
Cortelazzo 1999), del croato (Skok 1971-1974; Vinja 1998-2004), ed il Romanisches etymologisches Wörterbuch di Meyer-Lübke, del 1935, per l'etimo latino.

I verbi di cui è stata fatta l'analisi etimologica e lessicologica sono numerati e ordinati alfabeticamente. Ciascun lemma è accompagnato da frasi dalle quali la voce è stata estrapolata. Nella trasposizione dei lemmi sono state usate le trascrizioni fonologiche basate sull'alfabeto dell'Associazione Fonetica Internazionale (Dardano 2010). La spiegazione delle abbreviazioni usate per indicare i dizionari etimologici si trovano alla fine del lavoro.

3.1. Il lemma kapìti, v. "capire" entra nel ciacavo di Baratto attraverso l'istroveneto capir (DDV 99; VG 166; GDDT 127). L'etimologia remota della parola va cercata nel latino capere "capire" (DEI I/734; REW 1625; DELI 290), di origine indoeuropea.

- Homo u hižu prike će van biti zima ujče, kapite?

- Z Zajno se domislite ono ča je bilo kašnjije, ono ča je sada kapite malo težlje.

3.2. Il romanismo usato nel ciacavo di Baratto per esprimere il verbo "punire" è kaftigàtí. Deriva dal latino castigāre (REW 1746) originariamente "agire sulla purezza morale di qualcuno", denominale di castus "casto". Il termine è panromanzo. Alla diffusione del verbo ha contribuito l'uso ecclesiastico nel senso di "far penitenza, purificare" (DEI I/799; DELI 310). II termine entra nel ciacavo tramite l'istroveneto castigàr (VG 188; GDDT 136).

- Vidiš kako hi je buog kaštiga, tac ni stija dati meni vrta niš, sad su ga prodali.

3.3. La voce ciacava kumpanàt", v. "accompagnare" è un prestito dall'istroveneto compagnàr o cumpagnà (VG 236; GDDT 168; VDIP 86), accomodamento dall'italiano accompagnare. Si tratta di un composto parasintetico di compagno, che deriva dal latino tardo *companio (REW 2093), parola composta dal prefisso cum ed il sostantivo pānis, nel significato "chi mangia lo stesso pane" (DEI II/1034; SKOK II/235; DELI 366).

- (...) i smo ga kumpanjali poklje ja i tvoja teta, moja siestra Jana, na bruod valje u Genovu.

3.4. Il ciacavo kuntentàti, v. "contentare, far contento qualcuno" deriva da contentus (REW 2182; SKOK II/141). Secondo Cortelazzo/Zolli (DELI 385) contentus è il participio passato di continēre "contenere"; ovvero contento è chi si contiene entro limiti determinati. II lemma kuntentàti entra nel ciacavo di Baratto attraverso l'istroveneto contentàr (GDDT 171; VDIP 87; DDV 191). Rosamani (VG 281) dice che cuntènat è d'uso presso i bilingui dell'Istria interna.

- Uona je bila toliko kuntienta da je Vesna pozdravila (...)

- (...) kad bude vidija da si tuote uon će biti kuntenat.

3.5. Il termine usato nel ciacavo di Baratto per esprimere l'azione del "prendere" è $t$ fapàti, v. Si tratta di un prestito dall'istroveneto ciapàr, ciapà (VG 209; GDDT 148; VDIP 32). Secondo Skok (SKOK I/349) t Jap è un'onomatopea nostrana che esprime velocità e significa anche "gregge", da cui deriva t fapati. La voce deriva dal latino capulāre "accalappiare, allacciare", verbo denominale da capulum "cappio" (DEI II/892; JE I/113; REW 1666; DELI 329).

II passaggio della sibillante sorda $\mathrm{s}$ in sonora $\int$ davanti alle occlusive $\mathrm{k}-\mathrm{p}-\mathrm{t}$ è una caratteristica comune nel ciacavo (Sočanac 2004: 128).

$7 \quad$ È molto frequente il passaggio di o atona o tonica in u (Sočanac 2004: 119). 
- Se ženija za jenom z Žminja pa ga je ćapala z nikin drugin, pak ga je puštila.

- Sada je prodala suoje hiže je ćapala šjolde.

3.6. La forma dîgo viene presa dall'istroveneto e usata nel ciacavo nel significato di "dico; parlo di". Nei dizionari consultati questa forma ha funzione di interiezione o esclamazione con il significato di "ehi, dico!". Digo è la 1.p.sg. del verbo dir (DDV 192; VG 309; GDDT 200; JE I/127). Nella frase Ča dêla màti?...digo Stana? il termine digo viene inteso come "mi rifersico a, parlo di". Il verbo italiano dire deriva dal latino dícere (REW 2628), di origine indoeuropea, che in origine aveva il significato di "mostrare", quindi far conoscere per mezzo della parola (DEI II/1318; SKOK I/401; DELI 409).

3.7. Il termine ciacavo difturbàti, v. "disturbare" è una voce di origine dotta, dal latino disturbāre, da turbāre (DEI II/1360; REW 2696; DELI 484). Disturbäti è un prestito dall'istroveneto disturbàr (VG 322; GDDT 210).

- Ma ča si se dišturbala mi nositi, ča ću van ponuditi?

3.8. I lemma ciacavo fermäti, v. "fermare" deriva dal latino firmus (DELI 371; REW 3320; SKOK I/512; DEI III/1621), di origine indoeuropea. La voce passa nel ciacavo di Baratto tramite l'istroveneto fermàr (VG 368; GDDT 229; DDV 266).

- Mene po nuoći boli, po danu ne. - Ma ako niš ne delaš s njima ne, ku je muovin, ku je vako fiermo, pak me ne boli.

3.9. Il lessema fíniti, $v$. "finire" entra nel ciacavo di Baratto attraverso l'istroveneto finir (DDV 222; VG 379; GDDT 235). L'etimologia remota va cercata nel latino finìre (REW 3314), ovvero finis "fine" (REW 3315). Battisti/Alessio (DEI III/1647) indicano il significato di finis come "palo di confine". La forma è panromanza, ma non rumena.

- Sad će finiti uona, ma uona je mlajža dva lita, koliko ste reka?

- A baba je finila samo tri lita škuole?

3.10. Il lemma ciacavo gambîvati, v. "cambiare" è un prestito dall'istroveneto cambiàr o gambiàr (VG 421; GDDT 117; DDV 121). Deriva dal latino tardo cambiāre "barattare", di origine celtica (DELI 277; REW 1540; DEI I/698; JE I/167).

- (...) ni put se ni gambivalo ure kako i sada.

3.11. Il lemma metàti, v. "mettere" entra nel ciacavo di Baratto attraverso l'istroveneto mèter (DDV 550; VDIP 108) o mètar (VG 624; GDDT 375), continuatori del latino mittere "mandare" (REW 5616), evolutosi più tardi nel senso di "porre" (DEI IV/2445; DELI 973). Trattasi di forma indoeuropea e panromanza.

- A ma šoldi pasaju. - A znan. Ako hi uzmete skupa a hi ne mečete na kup...

- Čovik mi vajk meče pod nuos da uon...ma nismo svi jednaki.

3.12. II verbo muôvitị "muovere" deriva dal latino movēre (DELI 1017; REW 5703; SKOK II/461; DEI IV/2531). La forma passa al ciacavo di Baratto tramite l'istroveneto mòver (VG 655; GDDT 390; VDIP 112; DDV 430).

- Mene po nuoći boli, po danu ne. - Ma ako niš ne delaš s njima ne, ku je muovin, ku je vako fiermo, pak me ne boli. 
3.13. Il lemma ciacavo pârtiti, v. "partire", usato anche come eufemismo per "morire", è una voce dotta, prestata dall'istroveneto partir (DDV 410; VG 741; GDDT 436). II verbo deriva dal latino partire "dividere" (REW 6259), e poi intransitivamente "allontanarsi" (DEI IV/2785; DELI 1141), da pars,-tis.

- A lipo je pošlo njoj, nikad ni bila buona. - Nikad nanka knjižicu ni imala tamo. Moždani udar i gotovo, partila.

3.14. Skok (SKOK II/608) indica la presenza del lemma parăti, v. "parere, sembrare" nel ciacavo a partire dal Cinquecento, dal latino parēre "apparire" (REW 6235; DELI 1135). Il ciacavo parati o perati deriva dall'istroveneto parèr, (me par) (VG 739; DDV 407; GDDT 435).

- Ima samo jenu šćer ja paran.

- Sad znaš i tu novu. - A ne, to nisam znala, ja san perala da je uona tu.

- $\quad$ Piet lit? Ja san perala da ste bija manje u Taliji.

3.15. Il lemma ciacavo pasăti, v. "passare" è un prestito dall'istroveneto passàr o passà (DDV 412; VG 744; GDDT 437), che deriva dal latino volgare *passāre (REW 6267), da passus "passo" (DELI 1143; DEI IV/2792).

- Ma samo neka ta vrajža zima pasa.

- Ter i tuoj tac ni ku ne do trietega razreda, ja san do pietega pasa.

- Ja pasan poli njigovu hižu, kad gren h doktoru.

3.16. Il lessema ciacavo pensàti, v. "pensare" è un prestito dall'istroveneto pensàr o pensà (DDV 423; VG 759; GDDT 448). Trattasi di una voce colta derivata dal latino pē(n)sāre "pesare con cura" poi "giudicare" (DEI IV/2836; DELI 1163; REW 6391; JE III/17). Dalla stessa radice deriva anche il ciacavo spensâti se "inventare".

- Ja san pensala da nanka uona ni bila živa.

- $\quad$ Ča bimo još? Spensajse vrijeda.

3.17. Il ciacavo prontàti, v. "preparare" è preso dall'istroveneto prontàr (VG 831; DDV 537; GDDT 493). La voce deriva dal latino prōmptus "portato fuori" (REW 6776), participio passato di prōmere "tirar fuori", composto da prō "avanti" ed emere "comprare, prendere" (DELI 1270; SKOK III/50; JE III/89; DEI IV/3104).

- Kada je puodne opet gren dati kravan, svinjan, jančičan i poslje prontan užinu.

3.18. Il lemma ciacavo provàti, v. "provare" è un prestito dall'istroveneto provàr (DDV 468; VG 833; GDDT 494) che deriva dal latino probāre "riconoscere che una cosa è buona" (REW 6764; DEI IV/3121), da prōbus "di buona qualità" (DELI 1277). Si tratta di forma panromanza, ma non è presente nel rumeno.

- $\quad$ Ako vi provate, vi imate tablete na škrbelu, ku ne morete spati si popijete, ako ne ja ne je popijen.

- I uon je prova, sedan lit biti ćja brižan.

3.19. Il lessema regulàti, v. "ordinare, sistemare" passa al ciacavo di Baratto tramite l'istroveneto regolàr (VG 869; DDV 563). La voce deriva dal latino regula (REW 7177; DEI V/3224; SKOK III/125; DELI 1340).

- (...) smo si napisali zadaću i regulali i opet mati bi nas poslala za ovcami (...)

- Kad gren h maši si obučen brgeše, maju, jaketu, jeno malo bolje se regulamo. 
3.20. Il lemma ciacavo $\int p i ̂ d i t^{10}$, v. "spedire, mandare" deriva dal latino expedīre "sbrigare, preparare" (REW 3040; DELI 1582), formato dal prefisso ex-con il denominale di pēs "piede" (SKOK III/310; DEI V/3583). II lemma entra nel ciacavo di Baratto attraverso I'istroveneto spedir (VG 1067; VDIP 172; GDDT 601).

- A ča nisu mali uovce tamo. - Da? - A da i sve, sad kamo su no špidili ja neznan.

3.21. La voce Spjegàti, v. "spiegare" è un prestito dall'istroveneto spiegàr (VG 1071; GDDT 665; VDIP 174), continuazione del latino explicāre "svolgere, sciogliere", composto dal prefisso ex- e plicāre "piegare" (REW 3052; SKOK III/312; DEI V/3590; DELI 1587).

- Toni je hodija brati gljive, da Marica mu je rekla da su vrganji blizu kave, da će naj u jenoj boški, uona mu je špjegala kadi.

3.22. Il lessema ciacavo Sporkäti, v. "sporcare" deriva dal latino spurcus "impuro". La o aperta dell'italiano sporco si deve all'accostamento a porco (DELI 1593; REW 8194; SKOK III/410; DEI V/3600). La voce entra nel ciacavo di Baratto attraverso l'istroveneto sporcàr (VG 1078; GDDT 671; VDIP 176; DDV 694).

- $\quad$ (...) kad su bili Nijemci je bilo jako lipo, ali kad su bili Engleži, jako su bili šporki.

3.23. Il ciacavo Jtimàti, v. "stimare (riferito a persona)" è preso dall'istroveneto stimàr (DDV 631; VG 1093; GDDT 682), prestito dalla lingua letteraria, e deriva dal latino aestimāre "valutare" (REW 246; SKOK III/335; DEI V/3635).

- Tribe da me štimaš ku ćeš, ako ne nemuoraš!

- Mi je drago da ste došle vamo, donke ča ćeš ku ne duojti! Vero mi se štimajmo.

3.24. Il termine usato per esprimere il verbo "annoiare" nel ciacavo di Baratto è Stufäti, v. Deriva dal latino volgare *extūfāre "riscaldare" (REW 3108), parola composta dal prefisso ex- e un denominale del greco tŷphos "vapore" (DELI 1635; DEI V/3663). II termine entra nel ciacavo attraverso l'istroveneto stufàr "seccare, annoiare" (DDV 646; VG 1114; VDIP 182; GDDT 702), che si sviluppa dall'italiano stufare originariamente "lavare nella stufa".

- (...) smo hodili u Kanfanar u škuolu i smo se štufali hoditi priko Drage...

- $\quad$ Ste se štufale i tamo biti?

3.25. II ciacavo takàt, v. (o tekàt) "cominciare, stare accanto" deriva dall'istroveneto tacàr (GDDT 713; DDV 729; VG 1130; VDIP 198) o tacàt (VG 1131) "attaccare, appendere, essere attiguo, stare accanto, cominciare". Secondo Cortelazzo/Zolli (DELI 143) I'italiano attaccare non può essere stato ricavato da staccare con cambio di prefisso, come si ritiene nel DEI (1/352), siccome staccare è attestato molto più tardi. La voce quindi probabilmente deriva dall'antico taccare, con a-rafforzativo, nel significato di "apporre sui panni la tacca, ovvero la marca". II DELI non esclude la possibilità di una derivazione dal francese attacher, derivato a sua volta, con cambio di prefisso, dall'antico francese estachier "attaccare" ricavato da estache "piolo". Quest'ultimo deriva dal gotico *stakka "piolo" (REW 8218; JE III/250).

- Kada se hiže takivaju i ku uone prodadu, ča će uon? Muora i uon, ča će njemu to?

- (...) i tamo je teka stati u Arđentini.

- Drugog šetiembra kad teka škuola...

10 Il passaggio di $e$ in $i$ sia in posizione atona che tonica è frequente (Sočanac 2004: 120). 
3.26. La voce temperàti, v. deriva dal latino temperāre "moderare" (REW 8627), che in origine aveva il significato di "mischiare acqua e vino" (DEI V/3745; DELI 1675; SKOK III/457). Con lo stesso significato viene usato anche nel ciacavo di Baratto, ovvero "diluire qualsiasi bevanda". La voce è un prestito dall'istroveneto temperàr (VG 1145; DDV 741).

- Ćeš dati bocu, pa ćete temperati, ćete piti. Vina niman.

3.27. Il lemma ciacavo tiênditi ${ }^{11}, v$. "prendersi cura di" è preso dall'istroveneto tènder, nel significato di "badare, accudire" (VG 1146; DDV 742; VDIP 201). Secondo Doria tènder (GDDT 729) deriva dal latino attendere "attendere, prestare attenzione, prendersi cura di" (REW 763). Bisogna distinguere la voce dalla parola italiana tendere "dispiegare, protendere, porgere" che deriva dal latino tendere (REW 8640).

- (...) sada više krav nimamo jer nima mi ki tienditi.

- Bi vas bila forši tiendila bi, nikad neznate da li bi eli ne bi.

- Sad uona bi bila ništo dala svojoj šćeri do smrti, ku je bude tiendila, a nima niš!

3.28. II verbo ciacavo tornàti "tornare" è un prestito istroveneto, da tornàr (VG 1165; GDDT 746; DDV 758; VDIP 203). La voce deriva dal latino tornāre (REW 8794), che originariamente significava "lavorare al tornio", dal latino tornus "tornio", da qui si ha poi il significato di "muovere in giro, girare" (DELI 1709; DEI V/3830), per arrivare al significato attuale. II termine è panromanzo. Skok (SKOK III/525) fa poi risalire tornus al greco tórnos.

- (...) pokle je bija poša ča pak da će tornati nazad, vraga.

3.29. Il lessema ciacavo tukàti, v. "capitare, succedere" ha le sue origini nel latino medioevale toccāre, di origine oscura, probabilmente onomatopeica (*tokk-) secondo la comune opinione (DELI 1701; DEI V/3807). II termine tukäti entra nel ciacavo tramite I'istroveneto tocàr (DDV 679; GDDT 739; VDIP 203; VG 1159) o tucà (VG 1182), che significa anche "toccare".

- Kako je guorila uona mojoj materi "kuma Roža nemuojte dati dok ste vi živa, ud vas če puoj na vašu dicu, a z dice na vas nijeće", to je tukalo Mariju. - A da, to je tukalo njuoj!

3.30. Skok (SKOK III/530) indica che nel caso dei verbi ciacavi utprîti "aprire" e zaprîti "chiudere" abbiamo un incrocio di due radici, indoeuropea e protoslava. I corrispondenti croati dei due termini sono o(t)-tvoriti e za-tvoriti che presuppongono la radice *twer (DEI I/260). Nell'istroveneto si usano le voci aprì (VG 31; GDDT 32) o avrì (VG 50; DDV 52) e seràr (VG 1005; VDIP 157; GDDT 613) o chiùder/ciùder (VG 221; DDV 168). L'origine di aprìr è il latino aperïre (REW 515). Sembrerebbe che i termini ciacavi utprîti e zaprîti si siano formati entrambi da aprìr, con l'aggiunta dei prefissi croati $o(t)-, z a-$, do- e della desinenza -ti per l'infinito.

- Zapri vrata braće, da ni zima.

- Kad ni bilo tete doma, kad je delala, uona isto je došla. Ma smo bile zločeste, nismo je doprle.

- Mi imamo ključ, ma ne moremo se utpriti.

11 Il dittongamento della e lunga è frequente nel dialetto icavo-ciacavo. 


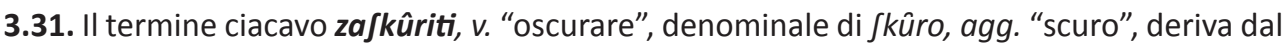
latino obscūrus (REW 6020), con aferesi della vocale iniziale (DELI 1490; SKOK III/274; $\mathrm{DEI} \mathrm{V} / 3431$ ). II termine è un composto ibrido formato dal prefisso croato za- e dal verbo deaggettivale Skuriti < Jkuro dall'istroveneto scùro (DDV 565; VG 990; VDIP 168; GDDT 606).

- Ma kako ste pa? - A kako, ča ja znan, ništo mi je forši zadilo. - Forši van se zaškurilo prid uoči?

3.32. Il lemma ciacavo zbaKäti"i , v. "sbagliare" è preso dall'istroveneto sbaliàr "sbagliare" (VG 940; GDDT 557). II termina deriva probabilmente, con cambiamento di prefisso, da abbagliare (DELI 1443), di etimo ancora discusso. L'origine più probabile è il greco baliós "macchiettato di bianco e di nero" o faliós "splendente, bianco", ovvero il latino volgare *balius (DEI I/405; JE III/326).

- Joh san rekla Roberto! San žbaljala!

\section{Conclusione}

Il contatto pluricentenario con Venezia ha lasciato un segno tangibile nei dialetti ciacavi dell'Istria. Con il presente lavoro lo si è voluto provare, analizzando etimologicamente trenta due verbi presi dalla parlata ciacava di Baratto di Canfanaro, zona interna rurale dell'Istria che ha risentito meno dell'influsso romanzo o veneziano, ma comunque contiene in sé tracce di tale contatto.

I verbi analizzati provengono tutti dall'istroveneto, che in Istria era lingua di prestigio e del commercio. I lemmi in questione hanno tutti origine latina, tranne takàt, evolutosi probabilmente da una voce francese, che trae poi le sue origini dal gotico. Sono presenti anche lessemi che nell'italiano sono grecismi (Stufati, zbaরati, tornati) e lemmi che derivano dal celtico (gambivati). I lemmi utpriti e zapriti sembrano formarsi da una base istroveneta (aprìr), con l'aggiunta di prefissi (ot-, za-, do-) e suffissi (-ti) ciacavi, adattandosi così alle regole morfologiche del ciacavo.

Nel passaggio dall'istroveneto al ciacavo i lessemi si adattano al sistema fonologico, morfologico e prosodico del ciacavo. I cambiamenti fonologici più comuni sono il passaggio di $s>\int$ davanti alle occlusive $k-p-t$; il passaggio di $o$ atona o tonica in $u$; il passaggio di $e>i$ sia in posizione atona che tonica; il passaggio di $z>3$. Sul piano morfologico, affinché una parola funzioni nel sistema linguistico ricevente, è obbligatoria la sostituzione dei morfemi legati. I verbi istroveneti di I coniugazione nel passaggio al ciacavo ricevono la desinenza -at(i), mentre quelli di II e III la desinenza -it(i), anche se ci sono delle eccezioni. Un altro cambiamento riguardante i prestiti verbali è quello dell'aspetto verbale, attraverso l'aggiunta dell'infisso - $v a$ - per indicare un'azione durativa o ripetitiva. I cambiamenti sul piano prosodico sono rari (finiti<finir; metati<mèter; partiti<partir; Spiditi<spedir), e riguardano lo spostamento dell'accento sulla sillaba precedente.

\section{Trascrizioni fonologiche}

/n/ (palatale nasale) campagna

/ts/ (dentale affricata sorda) calzolaio

/t $\int /$ (post-alveolare affricata sorda) cucina

12 È comune il passaggio della dentale $z$ in palatale 3 (Sočanac 2004: 129). 
/z/ (dentale fricativa sonora) sbagliare

$/ \mathrm{s} /$ (post-alveolare fricativa sorda) cuscino

$/ \mathrm{N}$ (palatale laterale) biglietto

/j/ (palatale approssimante) corriera

/3/ (post-alveolare fricativa sonora) fr. jour

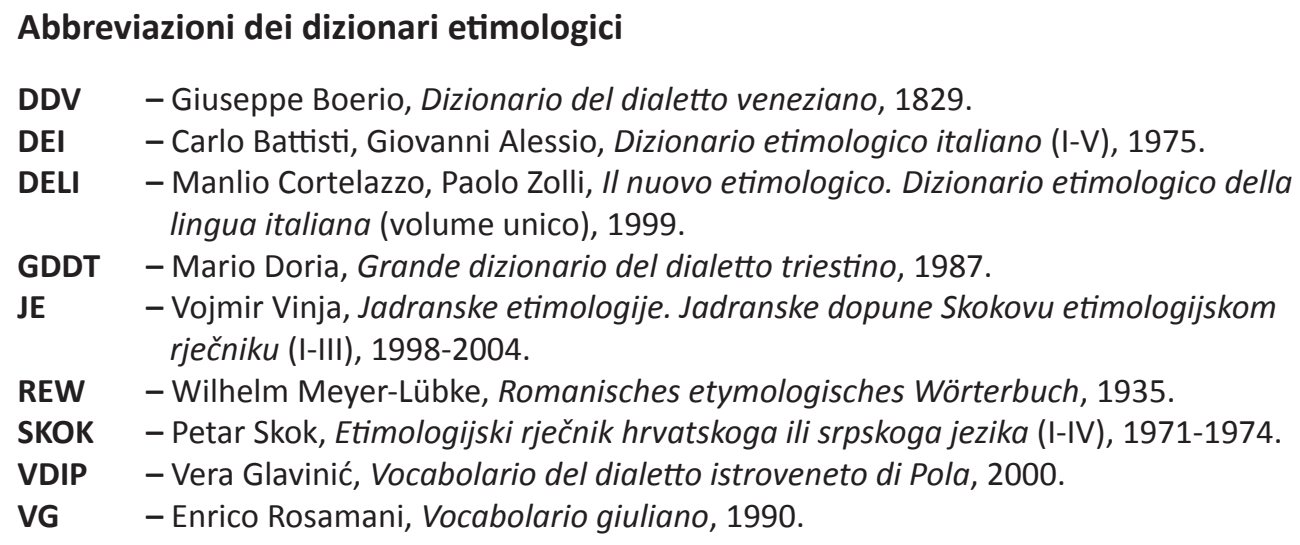

\section{RIFERIMENTI BIBLIOGRAFICI}

\section{BATTISTI 1975}

Carlo Battisti, Giovanni Alessio, Dizionario etimologico italiano (I-V), Firenze 1975.

\section{BERTOŠA 1995}

Miroslav Bertoša, Istra: "Doba Venecije (XVI.XVIII. stoljeće)", in: Povijest Istre 2, Pula 1995.

\section{BOERIO 1829}

Giuseppe Boerio, Dizionario del dialetto veneziano, Venezia 1829.

\section{BRATULIĆ 1985}

Josip Bratulić, Petar Šimunović (a cura di), Prezimena i naselja u Istri. Narodnosna statistika u godini oslobođenja I, Pula - Rijeka 1985.

\section{CORTELAZZO 1999}

Manlio Cortelazzo, Paolo Zolli, Il nuovo etimologico. Dizionario etimologico della lingua italiana, Bologna 1999.

\section{DARDANO 2010}

Maurizio Dardano, Pietro Trifone, La nuova grammatica della lingua italiana, Bologna 2010.

\section{DORIA 1987}

Mario Doria, Grande dizionario del dialetto triestino, Trieste 1987.

\section{FILIPI 1996}

Goran Filipi, "Dialettologia istriana", Scuola nostra 26, Fiume 1996., 113-121.

\section{FILIPOVIĆ 1986}

Rudolf Filipović, Teorija jezika u kontaktu, Zagreb 1986.

\section{GAČIĆ 1979}

Jasna Gačić, "Romanski elementi u splitskom čakavskom govoru", Čakavska rič 1, Split 1979., 3-54.

\section{GLAVINIĆ 2000}

Vera Glavinić, Vocabolario del dialetto istroveneto di Pola, Pula 2000.

\section{LISAC 2009}

Josip Lisac, "Čakavsko narječje”, in: Hrvatska dijalektologija 2, Zagreb 2009. 


\section{MEYER-LÜBKE 1935}

Wilhelm Meyer-Lübke, Romanisches etymologisches Wörterbuch, Heidelberg 1935.

\section{MULJAČIĆ 2003}

Žarko Muljačić, "O dvjema vrstama hrvatskih 'pseudoromanizama'", Filologija 40, Zagreb 2003., 95-112.

\section{PFISTER 2001}

Max Pfister, Antonio Lupis, Introduzione all'etimologia romanza, Catanzaro 2001.

\section{ROSAMANI 1990}

Enrico Rosamani, Vocabolario giuliano, Trieste 1990.

\section{SIMEON 1969}

Rikard Simeon, Enciklopedijski rječnik lingvističkih naziva II, Zagreb 1969., 317.

\section{SKOK 1971-1974}

Petar Skok, Etimologijski rječnik hrvatskoga ili srpskoga jezika (I-IV), Zagreb 1971-1974.

\section{SOČANAC 2001}

Lelija Sočanac, "Talijanski elementi u hrvatskom jeziku - kulturno i intimno posuđivanje", Riječ 17, n. 1, 2001., 77-88.

\section{SOČANAC 2002}

Lelija Sočanac, "Talijanizmi u hrvatskome jeziku", Suvremena lingvistika 53/54, n. 1/2, Zagreb 2002., 127-142.

\section{SOČANAC 2004}

Lelija Sočanac, Hrvatsko-talijanski jezični dodiri: s rječnikom talijanizama u standardnome hrvatskom jeziku i dubrovačkoj dramskoj književnosti, Zagreb 2004.

\section{TAMARO 2009}

Sandra Tamaro, "O bližnjoj i krajnjoj etimologiji romanizama iz semantičkog polja namještaja u govoru Boljuna", Čakavska rič 37, Split 2009., 105-121.

\section{VINJA 1998-2004}

Vojmir Vinja, Jadranske etimologije. Jadranske dopune Skokovu etimologijskom rječniku (I-III), Zagreb 1998-2004.

\section{WEINREICH 2008}

Ulrich Weinreich, Lingue in contatto, trad. Vincenzo Orioles, Torino 2008.

Sito internet dell'istituto nazionale di statistica (Državni zavod za statistiku), www.dzs.hr (25.10.2012) 


\section{RIASSUNTO}

Il lavoro è un'analisi etimologica dei prestiti verbali istroveneti presenti nella parlata ciacava di Baratto di Canfanaro. L'etimologia prossima di tutti i lessemi analizzati è stata individuata nell'istroveneto, mentre l'etimo remoto risale al latino. Alcuni lessemi traggono origine anche dal gotico, celtico o greco, oltre che dal latino. I cambiamenti subiti dai verbi sono avvenuti sul piano fonologico, morfologico, e in certi casi anche prosodico. L'analisi morfologica dei verbi ha mostrato la sostituzione delle desinenze istrovenete con quelle croate ciacave per un adattamento completo alla struttura grammaticale del ciacavo.

\section{Sažetak}

\section{Etimologija glagola istromletačkoga podrijetla u čakavskom govoru Barata (kraj Kanfanara)}

Rad predstavlja etimološku analizu istromletačkih glagolskih posuđenica u čakavskom govoru Barata kraj Kanfanara. Bliža etimologija svih obrađenih leksema utvrđena je u istromletačkom, dok krajnji etimon proizlazi iz latinskog. Pojedini leksemi, osim iz latinskog, vuku podrijetlo iz gotskog, keltskog ili grčkog. Glagolske promjene nastale su na fonološkoj, morfološkoj te u nekim slučajevima i prozodijskoj razini. Morfološka analiza glagola pokazala je zamjenu istromletačkih nastavaka s hrvatskim, odnosno čakavskim, kako bi došlo do potpune adaptacije glagola u čakavskom gramatičkom sustavu.

Ključne riječi: etimologija, romanizmi, čakavski, istromletački, Barat (Kanfanar) 